\title{
El debate actual sobre la(s) frontera(s) aplicado al caso ibérico: elementos de des-fronterización y re-fronterización entre España y Portugal en el siglo XXI
}

Rubén Camilo Lois González

Universidade de Santiago de Compostela

rubencamilo.lois@usc.es

Luis Alfonso Escudero Gómez

Universidad de Castilla-La Mancha

luisalfonso.escudero@uclm.es

Inês Gusman

Universidade de Santiago de Compostela

ines.gusman@gmail.com

Fecha recepción 13.07.2018 / Fecha aceptación 04.02.2019

\section{Resumen}

En las últimas décadas del siglo XX, los discursos sobre un mundo sin fronteras, donde las relaciones territoriales ya no son determinadas por los Estados, ganaron fuerza entre académicos y decisores políticos. Los proyectos de cooperación transnacional, como los que están en la base de la Unión Europea, parecían demostrar que las fronteras dejaban de significar discontinuidades económicas, políticas y sociales. Estos nuevos escenarios permitieron abrir una nueva fase en las relaciones entre territorios de España y Portugal y superar su

\begin{abstract}
Abstract: Since the last decades of the 20th century, the narrative of a world without borders, where territorial relations are no longer determined by States, has been gaining strength among academics and decision-makers. Transnational cooperation projects such as the European Union (EU) seem to demonstrate that borders no longer represent economic, political and social discontinuities. These new scenarios have opened a new phase in the relations between the territories of Spain and Portugal and overcome the historical
\end{abstract}


separación histórica. Este acercamiento se refleja en la creación de estructuras de cooperación transfronteriza y décadas de constante crecimiento de las relaciones económicas entre ambos países. Este proceso conoció un decaimiento durante la crisis económica iniciada en 2008. En este artículo, se analiza la evolución de estas relaciones y se estudia la efectividad de esta cooperación como marco adecuado para la superación de los efectos de la crisis. Entre los territorios portugueses y españoles, una vez superada la recesión, las relaciones transfronterizas retomaron su vigor, especialmente a nivel local y regional. Así, avanzando en las complementariedades culturales, sociales y económicas, la cooperación puede ir más allá de los límites de los Estados y puede concebirse como un instrumento efectivo de desarrollo territorial.

\section{Palabras clave}

Península Ibérica, cooperación regional, frontera, des-fronterización, re-fronterización. separation. This is a rapprochement reflected in the creation of cross-border cooperation structures and in the decades of constantly growing economic relations between both countries, although the process was slowed by the economic crisis beginning in 2008. In this paper, the focus is the evolution of these relationships and also an analysis of the effectiveness of cooperation as an adequate means for overcoming the effects of the crisis. Since the crisis ended, cross-border relations between Portugal and Spain have regained their vigour, especially at the local and regional levels. Thus, thanks to cultural, social and economic complementarities, cooperation can overcome the limits of States and be conceived as an effective instrument for territorial development.

\section{Keywords}

Iberian Peninsula, regional cooperation, border, des-bordering, re-bordering 


\section{Introducción}

El análisis de las fronteras ha preocupado a diferentes ciencias humanas y sociales. De hecho, esta dimensión multidisciplinar en su estudio es fundamental para aprehender toda la complejidad de los fenómenos fronterizos ${ }^{1}$.Las fronteras son líneas que separan territorios y a los individuos que residen en ellos. Por esta razón, adquieren múltiples dimensiones culturales, económicas, espaciales y sociales ${ }^{2}$. Las interpretaciones clásicas sobre las fronteras se han centrado en la evolución de los procesos en el tiempo. Desde la década de 1980, este análisis histórico está fijado en el principio de que las actuales fronteras son el resultado de la constitución de los Estados-nación ${ }^{3}$. Los Estados ya no responderían a una acumulación de evidencias seculares sobre la integración de pequeños lugares y regiones hasta formar un país completo. Por el contrario, se constituyen a través de ideas contemporáneas que condujeron a la creación de identidades nacionales «inventadas» en los distintos países y territorios nacionales.

Desde la Geografía, hay igualmente un interés principal sobre las fronteras. Se centra en los espacios y los territorios. De hecho, el estudio de las fronteras es una temática muy importante dentro de la disciplina, con destacados ejemplos tanto en la literatura francófona ${ }^{4}$ como en la anglófona ${ }^{5}$. Esta cuestión también ha preocupado a geógrafos ibéricos ${ }^{6}$, orientados estos en el estudio de la frontera entre España y Portugal ${ }^{7}$. La reflexión a través de los

1. J. M. Trillo Santamaría, Fronteras y regiones transfronterizas. El caso de Galicia-Norte de Portugal, tesis doctoral, 2010.

2. T. M. Wilson y H. Donnan, Border identities: Nation and state and international frontiers, Cambridge, 1998.

3. B. Anderson, Imagined communities: Reflections on the origin and spread of nationalism, Londres, 1983; E. Hobsbawn, The invention of tradition, Cambridge, 1983.

4. M. Foucher, Fronts et frontières. Un tour du monde géopolitique, Paris, 1991.

5. J. Sidaway, “Mapping border studies”, Geopolitics, 20, 2004, 214-222.

6. V. Cabero Diéguez, A. Campesino Fernández y L. López Trigal, "El conocimiento de las franjas fronterizas. Aportación de los geógrafos españoles”, Boletín de la Asociación de Geógrafos Españoles, 21-22, 1996, 83-96.

7. R. C. Lois González y A. Carballo Lomba, "La frontera hispano-lusa en la actualidad: una visión geográfica”, Revista de Historiografía, 23, 2015, 191-214.

Revista de historiografía 30, 2019, pp. 157-180 
tiempos históricos es imprescindible. No obstante, este artículo se desarrolla desde el análisis geográfico con una aproximación que se centra en el presente.

La definición tradicional de frontera la caracteriza como «líneas físicas y visibles de separación de espacios políticos, sociales y económicos ${ }^{8}$. Sin embargo, por su complejidad, las fronteras son más que simples líneas. Son el resultado de construcciones sociales complejas y por eso son elementos con diferentes significados y funciones 9 . A causa de ello, las formas de entender sus efectos en el espacio han cambiado a lo largo de los últimos años. De manera generalista, según los autores Newman ${ }^{10}$ o Kolossov ${ }^{11}$, hay tres grandes fases en el estudio de las fronteras en el siglo XX. La primera corresponde a aquellos estudios geopolíticos que incidían en la importancia de delimitar, demarcar y administrar correctamente las fronteras y los territorios. Esta etapa empieza a finales del siglo XIX y llega a mediados del siglo XX. Posteriormente, debido a las connotaciones negativas que adquiere la Geopolítica en los años 1950 derivadas de su manipulación política por regímenes autoritarios durante la Segunda Guerra Mundial, surgen hasta los años setenta y ochenta ópticas sobre la frontera diferentes. En este contexto, varios autores desarrollaron una mirada funcionalista, que enfocaba las funciones de las fronteras y los factores políticos y sociales que las determinaban ${ }^{12}$. La última fase empieza en la década de 1970 y se extiende hasta la actualidad. Está fuertemente marcada por la idea de interdependencia entre los territorios. Se han generado narrativas sobre la globalización que hicieron renacer los estudios sobre la frontera ${ }^{13}$.

El capitalismo contemporáneo, o capitalismo tardío, ha dado pie a la globalización en el último cuarto del siglo $\mathrm{XX}^{14}$. Aunque el proceso histórico de formación de un sistema global se puede extender al menos hasta el siglo XVI, Beck demuestra la novedad contemporánea que ha dado lugar la globalización desde las últimas décadas de la centuria pasada ${ }^{15}$. Ha supuesto transacciones comerciales allende las fronteras del Estado nacional al interior de un denso entramado con mayor dependencia y obligaciones recíprocas. Adquiere especial

8. D. Newman, "The lines that continue to separate us: borders in ourborderless' world", Progress in $\mathrm{Hu}$ man geography, 30, 2006, 143-161.

9. B. Haselsberger, "Decoding borders. Appreciating border impacts on space and people." Planning Theory \& Practice, 15, 2014, 505-526.

10. D. Newman. "On borders and power: A theoretical framework.", Journal of borderlands studies, 18, 2003, 13-25.

11. V. Kolossov, "Border studies: changing perspectives and theoretical approaches.", Geopolitics, 10, 2005, 606-632.

12. Kolossov, "Border studies...", op.cit., 608.

13. D. Newman y A. Paasi "Fences and neighbours in the postmodern world: boundary narratives in political geography", Progress in Human Geography, 22, 1998, 186-207.

14. D. D. Marshall, "Understanding late-twentieth-century capitalism: Reassessing the globalization theme", Government and Opposition, 31, 1996.

15. U. Beck, ¿Qué es la globalización? Falacias del globalismo, respuestas a la globalización, Barcelona, 1998 
importancia la globalización financiera ${ }^{16}$. Ha dado lugar a la «translocalización» de la comunidad, el trabajo y el capital. Ha creado una percepción de la transnacionalidad en los medios de comunicación, en el consumo y en el turismo. Finalmente, ha provocado una concentración económica y una nueva competencia de un mercado mundial que no conoce fronteras ${ }^{17}$.

En las últimas décadas, ha habido una pérdida de contenido de los Estados-nación frente a la integración supranacional y la descentralización. Los Estados-nación se ven vaciados de poder tanto desde instancias superiores, como el significativo caso de la Unión Europea (UE) y otras como Mercosur o el Tratado de Libre Comercio (NAFTA) ${ }^{18}$, como inferiores, a través de procesos de cesión de competencias y altos niveles de autogobierno a administraciones regionales y locales. Además, se multiplican los actores en la esfera internacional, más allá de los Estados, tanto públicos (regiones, ciudades, municipios) como privados (ONGs, asociaciones) ${ }^{19}$.

En el continente europeo se ha abierto paso a una imagen estatal unida ${ }^{20}$. Es un proceso político que ha recibido el neologismo anglófono de Europeanization. La eliminación de las fronteras nacionales dentro del espacio Schengen europeo y el creciente proceso de integración supranacional ha construido una Europa cada vez más unida. La obsolescencia de la función militar y administrativa en muchas de las fronteras europeas les ha hecho perder su sentido. No obstante, este proceso se realiza a diferentes velocidades, Irlanda permanece fuera del espacio Schengen y Bulgaria, Croacia, Chipre y Rumania no están todavía integrados; y no sin dificultades y retrocesos. El principal, la salida del Reino Unido de la UE tras el referéndum doméstico de 2016 que votó negativamente a la permanencia de este Estado en el citado organismo. Esta partida está en el momento actual aún pendiente.

Por otro lado, debemos significar que las fronteras todavía son fenómenos concretos cruciales para la organización espacial del mundo contemporáneo ${ }^{21}$. En la propia Europa, al tiempo que avanzaba la integración supranacional de la UE, los procesos derivados de la desaparición de los regímenes comunistas en el este y el colapso de la Unión Soviética supuso una re-fronterización mediante dinámicas de independencia, separación o reunificación ${ }^{22}$. En el presente, la frontera europea entre Ucrania y Rusia aún es motivo de un enfrentamiento militar activo. Además, ha habido un resurgimiento de identidades territoriales nacionalistas que, frente a la creencia historiográfica de finales del siglo pasado ${ }^{23}$, no han sido superadas. Es

16. Fondo Monetario Internacional (FMI), Financial globalization: The impact on trade, policy, labor, and capital flows, Washington, 2007.

17. U. Beck, ¿Qué es la globalización? ..., op. cit.

18. V. Konrad y H. Nicol, Beyond walls: Re-inventing the Canada-United States borderlands, Aldershot, 2008.

19. J. M. Trillo Santamaría y R.C. Lois González, "La frontera como motivo de atracción: una breve mirada a las relaciones Galicia-Região Norte", Geopolítica(s), 2, 2011, 109-134.

20. U. Beck, ¿Qué es la globalización? ..., op. cit.

21. A. Paasi, "Generations and the 'development' of border studies', Geopolitics, 10, 2005, 663-671.

22. M. Foucher, L’obsession des frontières, París, 2007.

23. E. Hobsbawn, Naciones y nacionalismo desde 1780, Barcelona, 1991.

Revista de historiografía 30, 2019, pp. 157-180 
el ejemplo del procés de Cataluña en España, que ha llevado a un grave enfrentamiento entre el gobierno autonómico y el central por el intento soberanista catalán.

En este artículo, se analizará el debate actual sobre las fronteras donde conviven procesos de des-fronterización con otros de re-fronterización. En las últimas décadas se han ido introduciendo los conceptos de des-fronterización o de-bordering ${ }^{24}$ y re-fronterización o re-bordering ${ }^{25}$ para intentar comprender las transformaciones que se vienen produciendo en las fronteras en el presente. Estos términos ya han sido aplicados en un trabajo previo sobre la Península Ibérica ${ }^{26}$.

El ámbito de estudio de la investigación se enmarca en la UE, que desde su creación trabaja por la integración entre sus Estados miembros y que ha impulsado y financiado la cooperación transfronteriza. Dentro de la UE, el estudio de caso que se desarrolla es el de España y Portugal. En concreto, y tras una breve introducción histórica, se entenderá cómo la entrada de los dos países ibéricos en la entonces Comunidad Económica Europea (CEE) en 1986 abrió una nueva fase donde la frontera política y militar ha perdido todo su significado. La des-fronterización hispano-lusa ha supuesto una ampliación y mejora de las relaciones entre ambos países en la esfera cultural, económica, social y territorial.

No obstante, las casi dos décadas que llevamos del presente siglo han sido convulsas por causa de un gran punto de inflexión, la crisis financiera iniciada en 2007 y convertida en recesión económica global tras la caída de la entidad financiera transnacional Lehman Brothers en septiembre de $2008^{27}$. Las terribles consecuencias socioeconómicas de la crisis se agravaron por distintas causas domésticas en España y Portugal tales como las malas prácticas financieras de sus bancos o el desarrollo de burbujas inmobiliarias. A partir de 2014, los grandes índices macroeconómicos de ambos países empezaron a mejorar y, con ello, se asume el principio del fin de la crisis (una salida más discutible en el plano social, aunque también aquí hay evidencias de superación, como la disminución de la tasa de desempleo). En nuestro objeto de estudio, la crisis supuso un fuerte declive de la cooperación transfronteriza. Sin embargo, como se analizará, en los últimos años ha habido ya un nuevo impulso y aparecen novedosos escenarios en las relaciones ibéricas. A raíz de esta recuperación, se abre para el futuro posibilidades de una mayor integración hispano-lusa que se plantean en el artículo. No obstante, la frontera no pierde su significado. Al tiempo que se reactiva la des-fronterización interna se vuelve a reforzar la re-fronterización peninsular con el exterior.

Los diferentes apartados que se desarrollarán se ajustan a la explicada evolución de la cooperación transfronteriza entre España y Portugal en el presente siglo. Con ello, este artí-

24. S. Stetter, "Regionalization and debordering: The Middle East between global interconnectivity and functional differentiation", en World society and the Middle East: reconstructions in regional politics, Basingstoke, 2008, 35-65.

25. K. S. Follis, "Introduction: Rebordering Europe", en Building fortress Europe: The Polish-Ukrainian frontier, Filadelfia, 2012, 1-25

26. M. Lois y H. Cairo, "Desfronterización y refronterización en la Península Ibérica”, Geopolítica(s). Revista de estudios sobre espacio y poder, 2, 2011, 11-22.

27. H. Dosdall y B.Z. Rom-Jensen, "Letting Lehman go: Critique, social change, and the demise of Lehman Brothers”, Historical Social Research, 42, 2017, 196-217. 
culo supone la continuación de una amplia línea de investigación seguida en estas temáticas por los autores. Los hallazgos y resultados de estos trabajos anteriores, sintetizados y revisados, son la estructura preliminar de este trabajo, junto a una exploración bibliográfica sobre la temática. La observación de los procesos actuales, con la utilización de fuentes en línea y periodísticas, y la reflexión analítica y deductiva dan lugar, finalmente, al núcleo fundamental del artículo: el estudio de los procesos de (des y re)-fronterización entre España y Portugal en el siglo XXI.

\section{La cuestión nacional en la construcción europea: Io nacional y lo fronterizo en la crisis y postcrisis}

La UE ha mantenido un objetivo constante de la desaparición de las fronteras internas. Así, esta institución ha implementado un conjunto de instrumentos políticos y económicos destinados a evitar las diferencias existentes entre los contextos nacionales para crear una especie de sociedad «supranacional» en Europa. Desde la firma del Tratado de Maastricht en 1992 y su entrada en vigor en 1993, se crea la actual UE, produciéndose una consolidación del mercado único y la libre circulación de mercancías, servicios y personas entre los Estados miembros ${ }^{28}$. Esto supone la desaparición de facto de las fronteras internas de la UE. Este hecho da lugar a nuevas dinámicas transfronterizas por Europa ${ }^{29}$. Se traduce en una transformación del papel de la frontera y una integración entre las territorialidades ${ }^{30}$. Se crean regiones transfronterizas, entendidas estas como el resultado de la interacción entre un espacio compartido a ambos lados de la frontera estatal y las acciones llevadas a cabo por los actores implicados en dicho contexto ${ }^{31}$.

Sin embargo, las políticas transfronterizas han demostrado ser insuficientes para originar la armonía entre las personas y las autoridades en cada lado de cada frontera de los 28 países de la $\mathrm{UE}^{32}$. Se trata de un proceso en construcción ${ }^{33}$. La mayoría de las regiones transfronterizas presentan aún una baja intensidad de cooperación ${ }^{34}$. En realidad, son muchos los proyectos de cooperación que se inician en gran parte gracias a los fondos comunitarios,

28. P. Murray y L. Holmes, Europe: Rethinking the boundaries, Aldershot, 2003.

29. A. L. Amilhat-Zsary y M. C., Fourny (Eds.), Après les frontières, avec la frontière, nouvelles dynamiques transfrontalières en Europe, La Tour d’Aigues, 2007.

30. Amilhat-Zsary y Fourny (Eds.), Après les frontières, avec la frontière..., op.cit., 12.

31. F. Zoido, S. De la Vega, G. Morales, R. Mas y R. C. Lois, Diccionario de Geografía Urbana, Urbanismo y Ordenación del Territorio, Barcelona, 2000.

32. R. C. Lois González, J. L. Palmeiro Piñeiro y M. Pazos Otón, "Integration, memory and cultural heritage in Galicia-Northern Portugal border region”, Nordia Geographical Publications, 36, 2007, 23-34.

33. F. López Palomeque, "Un nuevo mapa de Europa: conceptos, representaciones y percepciones del proceso de construcción política continental”, Polígonos: Revista de Geografía, 2003, 27-56.

34. X. Oliveras González, A. Durà Guimerà y M. Perkmann, "Las regiones transfronterizas: balance de la regionalización de la cooperación transfronteriza en Europa (1958-2007), Documents d’Anàlisi Geogràfica, $56,21-40$. 
pero no tantos los que logran altos niveles de integración ${ }^{35}$. Además, la crisis económica de 2008 ha supuesto un fuerte impacto en la cooperación transfronteriza, cuando no, en un primer golpe, su parálisis general.

En realidad, el objetivo federal de la UE y la desaparición de las fronteras se ha traducido en un proceso muy lento. En 1958 se creó, en el seno de la CEE, la primera eurorregión a lo largo de la frontera germano-holandesa ${ }^{36}$. Pero es, sobre todo, a partir de 1990 con la actual UE, cuando se han creado gran cantidad de regiones transfronterizas conocidas mayoritariamente como «Eurorregiones». Entre 1999 y 2007 se duplica la cifra acumulada en los cuarenta años precedentes ${ }^{37}$. Se trata de estructuras transfronterizas en las que, principalmente, las autoridades locales y regionales se han unido a través de una o varias fronteras nacionales. Han sido denominadas como «laboratorios de ensayo de la integración europea» ${ }^{38}$. Oliveras, Durà y Perkmann ${ }^{39}$ dividen la historia de la regionalización de la cooperación transfronteriza en Europa en tres grandes etapas: la primera, desde fines de la década de 1950 y hasta fines de la de 1970, serían los inicios de la cooperación transfronteriza, con hitos como la creación de la Asociación de Regiones Fronterizas de Europa (ARFE) en 197140; la segunda, entre finales de las décadas de 1970 y 1980, cuenta con los primeros instrumentos de apoyo legal como la Carta europea de las regiones fronterizas y transfronterizas de 1981 por parte de la ARFE; y la tercera, desde fines de los 1980 hasta 2007, se caracteriza por un impulso financiero, sobre todo con el programa Interreg ${ }^{41}$ iniciado en 1991, y por una fuerte expansión de las regiones transfronterizas.

La crisis económica global supuso la entrada de una cuarta etapa, con la paralización del proceso de cooperación transfronteriza, y el momento actual, con evidencias de recuperación económica a partir de 2014, plantea un nuevo escenario favorable para su reactivación. Sin embargo, otro grave problema oscurece hoy el proceso cooperativo entre los Estados miembros de la UE: la crisis de refugiados en Europa. En la UE, el principio de humanitarismo sincero obliga a acoger el flujo de desplazados. Sin embargo, la corriente masiva y constantes de refugiados, originada principalmente por la guerra civil en Siria, y la necesidad de coordinar los flujos de inmigrantes en el área de Schengen de fronteras internamente abiertas han creado una grave crisis en 2015 y se mantiene hasta la actualidad. Tanto más trágico es el bloqueo de las soluciones europeas por parte de Estados miembros poco

35. Trillo y Lois, "La frontera...”, op. cit., 129.

36. U. Wolf, A. Hollereder y H. Brand, "Cross-border cooperation in Europe. What are Euregios?", Gesundheitswesen, 68, 667-673.

37. Oliveras, Durà y Perkmann, "Las regiones transfronterizas..., op. cit.,23.

38. Wolf, Hollereder y Brand, “Cross-border cooperation..., op. cit., 667-673

39. Oliveras, Durà y Perkmann, "Las regiones transfronterizas...”, op. cit., 26.

40. Asociación de Regiones Fronterizas de Europa. En línea en: https://www.aebr.eu/en/index.php. [Consulta: 13.6 .18$]$.

41. Unión Europea, Interreg Europe. En línea en: https://www.interregeurope.eu/. [Consulta: 13.6.18]. 
inclinados a admitir a los refugiados, que están dispuestos a sacrificar la integración europea, porque no están listos para aceptar sus obligaciones humanitarias ${ }^{42}$.

\section{Un marco de cooperación exitosa hispanoportuguesa en la etapa del crecimiento, 1986-2008}

Historiadores portugueses llegan a situar la frontera entre España y Portugal en 1294 tras la firma del Tratado de Alcañices. Los datos históricos muestran que la idea de la línea fronteriza existe desde el siglo XIII, aunque la frontera política medieval no es una frontera completa, mental y social, tal como la entendemos hoy ${ }^{43}$. Esta frontera se confirmaría con la Guerra de la Restauración portuguesa a mediados del siglo XVII ${ }^{44}$. Sin embargo, esta delimitación no existe como línea hasta el establecimiento de los aparatos de estado moderno, un proceso que en Portugal y en España se retrasó ${ }^{45}$. De hecho, solo se trazó la frontera por la formación de unos Estados modernos deseosos de cobrar tributos, reclutar soldados y capaces de negociar y cartografiar una delimitación hasta entonces inexistente ${ }^{46}$. Es la firma del Tratado de Límites de Lisboa, en 1864, la que precisa finalmente y de una forma clara una frontera entre España y Portugal, aunque no sin problemas y conflictos, sobre todo entre los gobiernos centrales y las poblaciones locales ${ }^{47}$. De hecho, pasarán todavía cuatro décadas más hasta que en 1906 se firme el Acta General de Demarcación, que culminó desde el punto de vista legal y diplomático el acuerdo lisboeta ${ }^{48}$.

Se puede afirmar que durante siglos las regiones fronterizas constituyeron espacios de transición antes que un límite rígido. Por ejemplo, en la época moderna los intercambios fueron frecuentes en la frontera galaico-portuguesa ${ }^{49}$. La frontera delimitada en el tercer cuarto del siglo XIX solo funciona como tal durante el siglo $\mathrm{XX}^{50}$. Se produce entonces la militarización de la misma y se define una frontera en el sentido clásico del término. Este proceso

42. R. Baubock, "Europe's commitments and failures in the refugee crisis", European Political Science, 17, 2018, 140-150.

43. C. Barros, "La frontera medieval entre Galicia y Portugal", Medievalismo, 4, 1994, 27-39.

44. R.C. Lois González, "As relaçons de Portugal como a Ibéria: umaolhada desde a Galiza”, Lusotopie, 9, 2002, 193-208.

45. R. C. Lois González y J.I. Plaza Gutiérrez, "Development policies in the peripheral and marginal areas of the border between Spain and Portugal”, en International Geographical Union (IGU), 29th International Geographical Congress, Seúl, 2000, 317.

46. Lois, "As relaçons...", op. cit., 198.

47. H. Cairo y P. Godinho, "El Tratado de Lisboa de 1864: la demarcación de la frontera y las identificaciones nacionales", Historia y Política, 20, 2013, 23-54.

48. J. García Álvarez y P. Puente Lozano, "Las comisiones mixtas de límites y las representaciones geográficas de la frontera hispano-portuguesa (1855-1906)”, Revista de Historiografía, 2015, 67-100.

49. R.C. Lois González, "Galice-Portugal : des relations transnationales privilégiées dans la Péninsule Ibérique”, Sud-Ouest Européen, 18, 2004, 31-40.

50. Lois González, “Galice-Portugal..., op. cit., 31. 
tiene lugar durante el coincidente periodo de las dictaduras de Salazar, en Portugal, y Franco, en España ${ }^{51}$. Las dos fueron regímenes muy centralizados que no establecieron ninguna cooperación relevante ni apenas contacto, en realidad ${ }^{52}$. Sí que se mantuvo una movilidad transfronteriza de población, con personas que huían por razones como escapar al reclutamiento en Portugal durante la Guerra Colonial (1961-1974) o por motivos políticos durante la Guerra Civil española (1936-39) y la represión que le siguió ${ }^{53}$. También hubo una permeabilidad fronteriza económica a través del contrabando, pero las dictaduras permanecieron aisladas una de otra y los espacios fronterizos se consideraron como territorios marginales ${ }^{54}$.

En un territorio vecino, Galicia, y ya desde el autonomismo gallego de 1920-3055, hubo reivindicaciones por una relación prioritaria con Portugal ${ }^{56}$. Pensadores políticos abrieron una lectura de la realidad nacional de Galicia unida de forma natural a Portugal ${ }^{57}$. Sin embargo, de manera general, la Península Ibérica fue interpretada durante la mayor parte del siglo pasado como un espacio de desencuentro entre españoles y portugueses ${ }^{58}$, aunque con la destacable excepción de una tradición política iberista que en uno y otro país adquiere relieve desde el siglo XIX ${ }^{59}$. Esta situación cambia desde la incorporación de España y Portugal a la entonces CEE en 1986.

Así, la entrada de los dos países ibéricos en la CEE fue un gran impulso para las relaciones bilaterales. La comunidad europea suponía un marco propicio para el reencuentro. España y Portugal pasan a estar integrados plenamente en Europa. Gracias a las políticas de cooperación, la frontera deja de ser un motivo de repulsión y cierre. Se transforma en apertura y contacto. Evoluciona de una barrera a un puente ${ }^{60}$. De la separación se pasa a la cooperación transfronteriza ${ }^{61}$. Por lo tanto, desaparece la frontera entre España y Portugal con la integración europea, al menos en las limitaciones del tránsito de personas y de mercancías. Un proceso que se repite en la otra gran frontera de la Península Ibérica, la franco-española ${ }^{62}$.

\footnotetext{
51. Lois, “Galice-Portugal..., op. cit., 33.

52. Lois y Carballo, “La frontera hispano-lusa..., op. cit., 196.

53. Lois y Carballo, “La frontera hispano-lusa..., op. cit., 201.

54. Lois y Carballo, "La frontera hispano-lusa..., op. cit., 197.

55. Lois, “Galice-Portugal..., op. cit., 35.

56. El pensador Castelao reclamaba una «República Federal Hespañola para confederarse com Portugal», en A. R. Castelao, Sempre en Galiza, Belgrano, 1961, 477.

57. X. Beramendi, De Provincia a Nación. Historia do galleguismo político, 2008, Vigo.

58. Lois, “As relaçons...”, op. cit., 193-208.

59. J. A. Rocamora Rocamora, "Un nacionalismo fracasado: el iberismo", Espacio, Tiempo y Forma, Serie V, Historia Contemporánea, 2, 1989, 29-56.

60. Trillo y Lois, “La frontera..., op. cit., 109.

61. C. Cancela (Coord.), Cooperación transfronteriza: comparando las experiencias ibéricas, Santiago de Compostela, 2008.

62. J. B. Harguindéguy, La frontière en Europe : un territoire? Coopération transfrontalière franco-espagnole, París, 2007.
} 
La eliminación de fronteras y la integración económica impulsó el comercio y la inversión exterior directa. De esta forma, se crearon grandes grupos empresariales que operan en las dos naciones de forma indiferenciada en sectores como el cemento, la madera o el textil ${ }^{63}$. Al mismo tiempo, el valor del comercio exterior entre Portugal y España aumentó cuantitativamente, tal y como se ha demostrado para el caso gallego y el país vecino desde la década de $1990^{64}$. Complementariamente, algunas pequeñas y medias ciudades fronterizas portuguesas se han especializado en el aprovisionamiento de infraestructuras industriales al servicio de las empresas españolas. Es el caso de Vilanova de Cerveira y Valença, núcleos que han promovido la construcción de centros industriales para atraer la inversión desde Galicia. Al mismo tiempo, surgen experiencias conjuntas de desarrollo local y un mejor conocimiento de los responsables públicos de uno y de otro lado de la frontera, entre otros efectos ${ }^{65}$. Hay una eclosión de proyectos transfronterizos de cooperación ${ }^{66}$.

Además, desde 1986 considerables volúmenes de fondos europeos de cooperación transfronteriza dan lugar a mejoras sustanciales en las infraestructuras de comunicación. Se construyeron importantes puentes fronterizos: en 1991 sobre el Guadiana, entre la localidad onubense de Ayamonte y Castro Marim en el Algarve portugués, y en 1995 sobre el Miño, entre el núcleo gallego de Salvaterra do Miño y el luso de Monção. Facilitan una movilidad transfronteriza que hasta entonces era muy complicada ${ }^{67}$.

Sin embargo, no en todos los aspectos se ha progresado en la cooperación transfronteriza. Hay notables excepciones donde ha priorizado la competencia entre ciudades antes que cualquier lógica económica y espacial en la red de comunicaciones. Sucede en los sistemas aeroportuario y portuario, especialmente en la eurorregión Galicia-Norte de Portugal. Aquí, se contabilizan cuatro aeropuertos, tres en Galicia y el de Sá Carneiro en Oporto. La concurrencia ha sido calificada como nefasta ${ }^{68}$. Se detecta una excesiva oferta que repercute en el margen de crecimiento y la rentabilidad de los aeropuertos. Una concentración sería deseable, pero difícilmente alcanzable por los intereses de cada ciudad aeroportuaria. En el caso del transporte marítimo, también existe un elevado número de puertos de alta capacidad en un espacio limitado (Ferrol, A Coruña, Vilagarcía, Marín y Vigo, en Galicia, y Viana do Castelo y Oporto-Leixões, en la Región Norte de Portugal). Si estos puertos pudieran formar uno solo, con múltiples terminales, sería una infraestructura muy competitiva capaz de captar el creciente flujo de tráfico que se dirige hoy a los puertos del noroeste europeo ${ }^{69}$. También en este caso la coordinación parece muy lejana en el presente.

63. R.C. Lois González, "Galiza no espaço lusófono. Uma aproximação a partir da Geografia”, Revista de Geografía e Ordenamento do Territorio, 10, 2016, pp. 9-33.

64. Lois, "Galice-Portugal...", op. cit., 35-36.

65. A. Meixide y A. de Castro (Coords.), Galicia e a Rexión Norte de Portugal: un espacio económico europeo, A Coruña, 2001.

66. L. López Trigal y F. Guichard (Eds.), La frontera hispano-portuguesa: nuevos espacios de atracción y cooperación, Zamora, 2000.

67. J. M. Jurado Almonte (Dir.), El turismo en el Arco Suratlántico onubense-algarví, Huelva, 2001.

68. Lois, “Galice-Portugal..., op. cit., 38.

69. Lois, “Galice-Portugal..., op. cit., 38.

Revista de historiografía 30, 2019, pp. 157-180 
Igualmente, se ha de tener en cuenta en este proceso de des-fronterización hispanoportuguesa un hecho diferenciador importante. Los espacios fronterizos entre España y Portugal no son homogéneos. Una división clásica es la establecida entre la frontera (o raya ${ }^{70}$ ) rica y la pobre ${ }^{71}$. La primera, la también denominada «raya húmeda» ${ }^{72}$, se establece con el río Miño, entre Galicia y el distrito portugués de Viana do Castelo, y con el Guadiana, entre Huelva (Andalucía occidental) y el Algarve. Son espacios con elevadas densidades demográficas, una intensa urbanización y claras complementariedades económicas. Por el contrario, la mayor parte de la antigua frontera es pobre, periférica y manifiesta un declive aún no corregido. Es la «raya seca» ${ }^{73}$ que abarca el resto de la frontera entre los dos países.

Por otro lado, entre España y Portugal existe también una frontera oceánica exterior. Es la delimitada en el Atlántico entre las españolas Islas Canarias y el archipiélago portugués de Madeira. Entre ambos archipiélagos hay contactos humanos y comerciales desde el siglo $\mathrm{XVI}^{74}$. Actualmente, se delimitan sus espacios marítimos de acuerdo al Derecho Internacional del Mar y, en concreto, según la Convención de la ONU de 1982, firmada por ambos Estados $^{75}$. Se han establecido así dos zonas económicas, portuguesa al norte y española al sur. Sin embargo, no es una frontera libre de conflictos dado que unos pequeños islotes intermedios, conocidos como Islas Salvajes, generan tensiones diplomáticas entre ambos países y permanecen como una zona de discordia ${ }^{76}$. Al mismo tiempo, las Canarias, Madeira y también el otro archipiélago portugués atlántico, las Islas Azores, son consideradas como regiones ultraperiféricas dentro de la $\mathrm{UE}^{77}$. Esta situación les permite regulaciones específicas, más desarrolladas en el caso de Canarias, comunidad autónoma que tiene un régimen económico y fiscal propio dentro de España. Los archipiélagos atlánticos ibéricos han sido objeto de colaboración transnacional en el marco de la Cooperación Territorial Europea. De hecho, en 2007 la Comisión Europea aprobó el «Programa de Cooperación Transnacional Madeira-Azores-Canarias (MAC) 2007-2013», con el fin de impulsar el desarrollo de un espacio común de crecimiento e integración económica, social y cultural ${ }^{78}$.

70. L. López Trigal, R. C. Lois González y F. Guichard (Eds.), La articulación territorial de la raya hispanoportuguesa, Zamora, 1997.

71. Lois, "As relaçons...", op. cit., 197.

72. Lois y Carballo, "La frontera hispano-lusa..., op. cit., 194.

73. Lois, "As relaçons...”, op. cit., 197.

74. M. Lobo Cabrera, "Gran Canaria y los contactos con las islas portuguesas atlánticas: Azores, Madera, Cabo Verde y Santo Tomé”, en F. Morales (Coord.), V Coloquio de Historia Canario-Americano, Las Palmas de Gran Canaria, 1982, 312-333.

75. J. M. Lacleta Muñoz, "Las fronteras de España en el mar", Boletín Elcano, 47, 2005, 17.

76. A. Sereno, "El nuevo mapa marítimo de Portugal y el caso de las Islas Salvajes", Revista Electrónica de Estudios Internacionales, 28, 2014, 1-29.

77. A. Brito Pérez, "Las regiones ultraperiféricas de la Unión. Una dimensión singular. Especial referencia a Canarias", Revista de Derecho de la Unión Europea, 12, 2007, 161-194.

78. Comisión Europea, “Cooperación territorial transnacional 'Madeira-Azores-Canarias". En línea en: https://ec.europa.eu/regional_policy/es/atlas/programmes/2007-2013/crossborder/operational-programmemadeira-acores-canarias. [Consulta: 21.02.19]. 
Retornando a la frontera peninsular, objeto principal de la investigación, se pueden establecer distintos grados de relación. Así, entre Castilla y Portugal, la existencia de contrastes culturales entre áreas rurales despobladas define un límite muy claro. Por el contrario, entre Extremadura y el Alentejo y entre Andalucía y el Alentejo o el Algarve, los intercambios y los intereses comunes son mucho más grandes. En la parte septentrional, entre Galicia y la Región Norte de Portugal, la frontera es poco significativa y las afinidades territoriales de la fachada atlántica ibérica se han reforzado en las últimas décadas ${ }^{79}$. Es aquí donde el proceso de des-fronterización ha sido más intenso. En 2015, de los 69.000 vehículos que cruzaron la frontera portuguesa en ambos sentidos, 33.000 lo hicieron a través de los pasos gallegos ${ }^{80}$. Galicia es la comunidad autónoma con más peso en las exportaciones de Portugal a España ${ }^{81}$

\section{El declive de la cooperación transfronteriza durante la crisis, 2008-2014}

En los periodos de crisis, se produce un reforzamiento del papel de la frontera, muchas veces inducido o ratificado por decisiones políticas ${ }^{82}$. Así ha sucedido en la reciente recesión económica que España y Portugal han sufrido intensamente entre 2008 y 2014. La integración europea y, en concreto, la cooperación transfronteriza venía recibiendo un impulso clave por parte de la UE mediante las iniciativas comunitarias de fondos estructurales, de carácter financiero. Sucedía fundamentalmente a través del programa Interreg ${ }^{83}$. De hecho, en el periodo de programación previsto para 2007-2013, la explícitamente denominada Cooperación Territorial Europea era un objetivo principal de la política regional ${ }^{84}$.

Los acontecimientos dieron lugar a una realidad distinta a la planificada. Los capitales disponibles se acabaron dirigiendo mayoritariamente a la respuesta ante la crisis financiera y económica de 2008. Los fondos de la UE se dedicaron a intentar paliar y solventar los efectos de la crisis. Principalmente, se procuró estabilizar la moneda única europea, el euro, sostener a las entidades financieras de los países miembros y rescatar, hasta varias veces en algunos casos, la economía general de los Estados más afectados. La UE acabó asumiendo parte de la deuda soberana que estos Estados adquirieron para evitar, a su vez, la quiebra de sus bancos domésticos e intentar solventar su crisis económica nacional.

Como consecuencia de toda esta coyuntura, los presupuestos de la UE establecidos con anterioridad a la crisis fueron drásticamente modificados y las partidas planificadas mayo-

79. Lois, “Galice-Portugal..., op. cit., 32.

80. Ministerio de Fomento (2017), “Observatorio transfronterizo España-Portugal (OTEP)”. En línea en: https://www.fomento.gob.es/MFOM.CP.Web/handlers/pdfhandler.ashx?idpub=BTW032. [Consulta: 3.07.18].

81. L. Pinto, "Galiza aumenta pressão sobre indústria nortenha”. En línea en: https://www.publico. pt/2018/01/29/economia/noticia/galiza-aumenta-pressao-sobre-industria-nortenha-1800964. [Consulta: 19.06.2018].

82. Lois y Caraballo, "La frontera hispano-lusa..., op. cit., 193.

83. Oliveras, Durà y Perkmann, "Las regiones transfronterizas..., op. cit., 27.

84. Trillo y Lois, “La frontera...”, op. cit., 115. 
ritariamente recortadas. Así sucedió con los fondos destinados a la Cooperación Territorial Europea. Esto conllevó la práctica suspensión de los proyectos conjuntos que estaban contemplados en el programa Interreg para el periodo 2007-2013 $3^{85}$

Por otro lado, España y Portugal han recurrido a políticas nacionalistas propias para buscar una «solución» a la crisis, si bien influidas claramente por las directrices dictadas por la Comisión Europea, el Banco Central Europeo y el Fondo Monetario Internacional (en ámbitos mediáticos, pero, también, académicos la denominada Troika $a^{86}$ ). Ambos países se han visto especialmente afectados por la recesión económico-financiera que comenzaba en 2008. Las medidas adoptadas tienen claros puntos en común como la denominada política de austeridad, que reduce los presupuestos públicos en servicios sociales, entre otras decisiones, y las reformas laborales. Sin embargo, España y Portugal no han buscado una cooperación ibérica conjunta para la recuperación. Al contrario, el número de acuerdos alcanzados en las cumbres bilaterales hispano-portuguesas se redujo ${ }^{87}$. Los dos Estados siguieron programas similares determinados por la Troika, pero de una forma independiente entre sí. Incluso socialmente, las protestas que los «indignados» ${ }^{88}$ realizaban en España coincidían en el tiempo con las huelgas y manifestaciones en Portugal ${ }^{89}$ sin que, realmente, hubiese ninguna relación importante entre ambos movimientos.

La recesión ha supuesto un descenso de la actividad económica y, como es lógico, un decrecimiento de los flujos entre España y Portugal (fig.1). El comercio y la inversión exterior se reducen. También influyó la profunda crisis de determinados sectores, como la construcción; el hundimiento de empresas emblemáticas, como la agroalimentaria Pescanova, y la disminución del consumo. Todo ello ha dado lugar a una disminución en los movimientos de capital transfronterizos ${ }^{90}$.

En esta situación de recesión económica, se ha hecho evidente la fragilidad de ciertas dinámicas de movilidad. Es el caso de los movimientos laborales, en especial las relaciones empresariales, y los derivados del consumo. Se ven gravemente afectados por la paralización económica asociada a la crisis y sus consecuencias, como el elevado aumento del desempleo en los dos Estados ibéricos, superior en España que en Portugal. El incremento

\footnotetext{
85. Unión Europea, Interreg IVC. En línea en: http://www.interreg4c.eu/. [Consulta: 13.06.18].

86 .S. Cohen, M. D. Guillamón. I. Lapsley y G. Robbins, "Accounting for austerity: the Troika in the Eurozone”, Accounting, Auditing \& Accountability Journal, 26, 2015, 966-992; R. Méndez, S. Sánchez-Moral y J. Malfeito-Gaviro, "Employment changes in knowledge-based industries in large urban areas of Spain: Impact of the economic crisis and austerity policies", Environment and Planning C: Politics and Space, 34,2015 963-990; C. Moury y A. Standring, “'Going beyond the Troika': Power and discourse in Portuguese austerity politics”, European Journal of Political Research, 56, 2017, 660-679.

87. Lois y Carballo, "La frontera hispano-lusa..., op. cit., 212.

88. E. Romanos, "Late neoliberalism and its indignados: Contention in austerity Spain”, enLate neoliberalism and its discontents in the economic crisis. En línea en: https://ink.springer.com/chapter/10.1007\% 2F978-3-319-35080-6_5. [Consulta: 16.6.18]

89. G. Accornero, “Mild mannered'? Protest and mobilisation in Portugal under austerity, 2010-2013”, West European Politics, 38, 2014, 491-515.

90. Lois, “Galiza no espaço lusófono...”, op. cit., 22-23.
} 


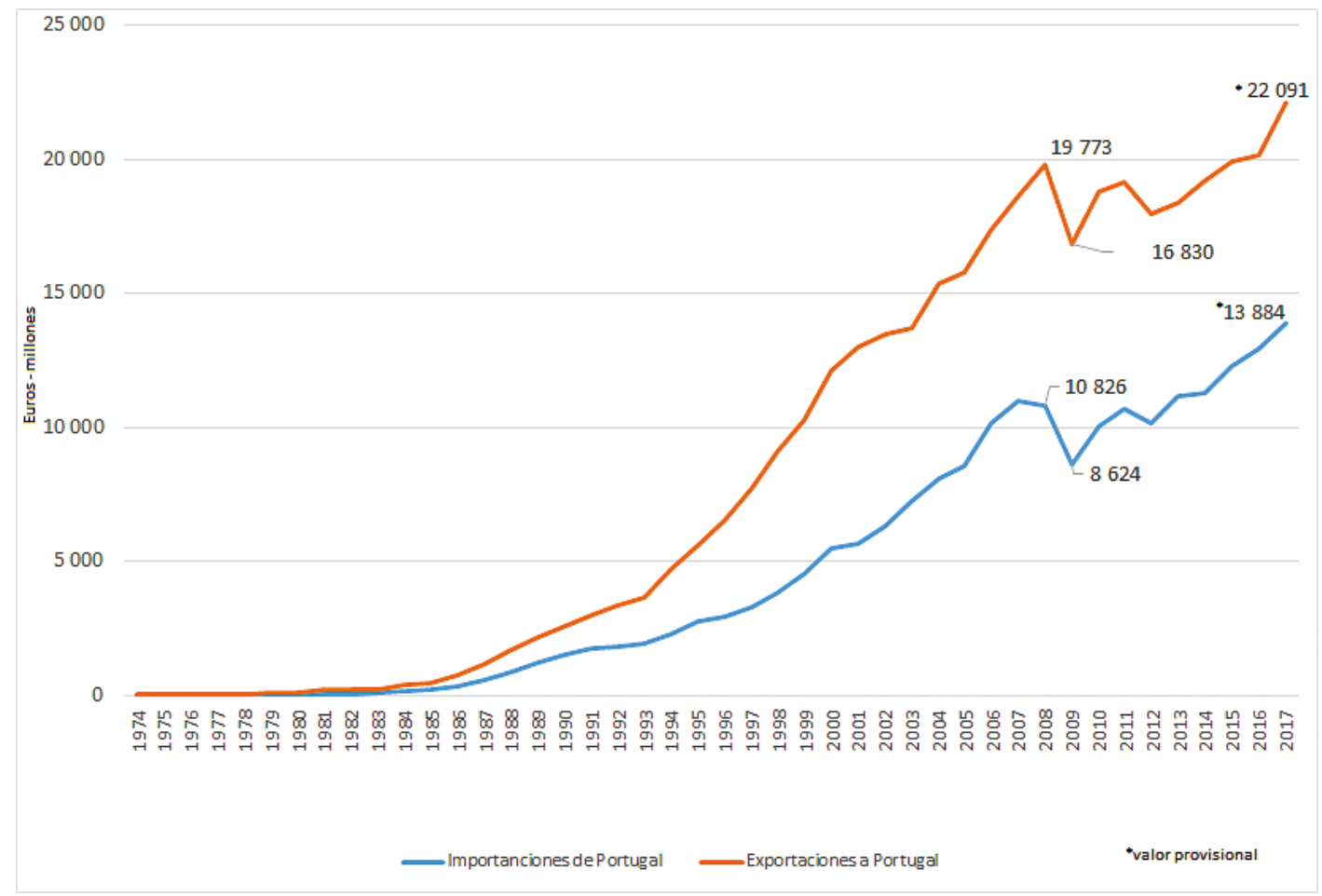

Figura 1. Flujos comerciales entre España y Portugal 1974 y 2017.

Fuente: Elaboración propia a partir de PORDATA (2018).

del paro redujo el número de contrataciones de trabajadores del otro país. Además, disminuyeron los gastos dedicados al ocio y al turismo, lo que también influyó en la reducción de los contactos. En consecuencia, en la movilidad de la población aparece una clara contracción en comparación con las épocas de crecimiento económico ${ }^{91}$. Además, el gobierno portugués impuso una medida recaudadora, entre otras muchas, con el fin de la recuperación económica y que ha afectado a la movilidad transfronteriza. Es la tasa que grava el tránsito por las autovías portuguesas. Consiste en un proceso telepeaje que elevó el coste de la movilidad. A mayores, el procedimiento de pago resulta complejo para un usuario eventual. Esta medida ha sido calificada como la decisión gubernamental portuguesa anticrisis más lesiva para la cooperación transfronteriza ${ }^{92}$.

91. Lois, “Galiza no espaçolusófono..., op. cit., 24.

92. Lois y Carballo, “La frontera hispano-lusa..., op. cit., 214. 
Una cuestión más a considerar sobre los efectos de la crisis es la parálisis del proyecto ferroviario común. La integración transfronteriza a través de las infraestructuras de comunicación tenía el desafío del tren de gran velocidad. Los retrasos con respecto a lo planificado ya eran evidentes antes de la crisis ${ }^{93}$. La recesión económica ha paralizado este gran proyecto y todas las conexiones diseñadas entre España y Portugal parecen, hoy en día, lejanas. Los enlaces previstos entre Lisboa y Madrid o Oporto y Vigo están paralizados sin fecha prevista para retomarlos ${ }^{94}$. En Portugal, los proyectos de un ferrocarril de alta velocidad están totalmente suspendidos ${ }^{95}$.

A pesar de que la crisis supuso un importante freno a la creciente cooperación transfronteriza hispano-lusa, se siguieron manteniendo los contactos y surgieron algunas experiencias comunes en la frontera. Es el caso de las eurociudades. Se constituyen a partir del deseo de dos localidades fronterizas de afrontar una gobernanza común en determinados servicios, en actividades culturales y en promoción turística al tiempo que desarrollan proyectos comunes de desarrollo local ${ }^{96}$. Han nacido en los años de recesión y son un claro ejemplo del mantenimiento de la cooperación transfronteriza, más allá de la paralización financiera. Así, la eurociudad de Chaves-Verín se creó en 2008, en 2012 la de Tui-Valença do Minho y en 2013, fuera del estrecho ámbito de relación galaico-portugués, se constituyen la de Badajoz-Elvas y la de Ayamonte-Vila Real de Santo António. Tras estas primeras experiencias, se ha creado una quinta eurociudad en 2015, la tercera en la raya entre Galicia y Portugal, entre las dos localidades unidas por el puente internacional sobre el río Miño del año 1995, Salvaterra do Miño y Monção.

Otra interesante experiencia transfronteriza en tiempos de crisis tiene lugar en el ámbito universitario. Es el programa IACOBUS. Se desarrolla en la eurorregión de Galicia-Norte de Portugal y está firmado por sus universidades y centros de enseñanza superior. Su objetivo es promover el desarrollo de la enseñanza superior y la investigación científica y tecnológica conjunta en la eurorregión ${ }^{97}$. Con este programa se ha animado la movilidad de varios cientos de profesores, investigadores y funcionarios universitarios. Por cierto, las estancias concedidas a miembros de las universidades de A Coruña, Santiago de Compostela y Vigo duplican a las de los portugueses procedentes de los politécnicos y universidades públicas de la Región Norte $^{98}$, lo que sigue reflejando una desigualdad de comportamiento frente a la cooperación transfronteriza (Galicia percibe más cerca a Portugal, que a la inversa).

93. Lois, “Galice-Portugal...”, op. cit., 38.

94. Lois y Carballo, “La frontera hispano-lusa...", op. cit., 212.

95. O. Igea, "Portugal entierra el AVE”. En línea en: http://www.diariovasco.com/sociedad/201502/06/ portugal-entierra-201502061022.html. [Consulta: 15.06.18].

96. M. Lois Barrio, "Re-significando la frontera: el caso de la eurociudad Chaves-Verín", Boletín de la Asociación de Geógrafos Españoles, 61, 2013, 309-328.

97. Xunta de Galicia, Programa Iacobus. En línea en: http://iacobus.gnpaect.eu/es/programa-iacobus. [Consulta: 16.06.18].

98. Lois, “Galiza no espaço lusófono..., op. cit., 28. 


\section{Un nuevo impulso a la cooperación: los programas POPTEC y la cumbre de Vila Real}

Desde el año 2014, superada la recesión más profunda derivada de la crisis global de 2008, se ha reactivado la cooperación transfronteriza. Así, se ha retomado el programa Interreg y sus fondos. La UE destina 359 millones de euros, financiados a través del Fondo Europeo de Desarrollo Regional (FEDER), para el período de 2014-2020. Estas ayudas se dirigen a gobiernos locales y regionales europeos para «(...) desarrollar y ofrecer mejores políticas. Al crear un entorno y oportunidades para compartir soluciones, nuestro objetivo es garantizar que la inversión del gobierno, la innovación y los esfuerzos de implementación conduzcan a un impacto integrado y sostenible para las personas y el lugar» ${ }^{99}$. Más allá de la literatura institucional habitual del organismo europeo, está el hecho de la disponibilidad de fondos para proyectos de cooperación interregional, con una financiación de hasta el $85 \%$ de los $\operatorname{mismos}^{100}$. Es decir, se supera la suspensión de la financiación y se reactivan fondos para la cooperación.

Con los capitales de Interreg se ha puesto en marcha el Programa de Cooperación Transfronteriza España-Portugal (POCTEP) para el periodo 2014-2020101. En su primera convocatoria, se han aprobado 132 proyectos de cooperación de los 456 presentados con una inversión total de 237,97 millones de euros, 1.782,28 millones provenientes del FEDER de la UE ${ }^{102}$. El POCTEP 2014-2020 actúa en cinco grandes ámbitos u objetivos temáticos: potenciar la investigación, el desarrollo tecnológico y la innovación; mejorar la competitividad de las pequeñas y medianas empresas; promover la adaptación al cambio climático en todos los sectores; proteger el medio ambiente y promover la eficiencia de los recursos; y mejorar la capacidad institucional y eficiencia de la administración pública ${ }^{103}$.

Sin embargo, entre los proyectos elegidos se detecta la repetición de un error, el de la financiación encubierta. En un repaso a la lista, superior a la centena, de proyectos aprobados aparecen muchos de carácter sectorial (desarrollar las tecnologías en el sector naval y metalmecánico, favorecer el cultivo del olivo, etc.) o muy especializados (investigación en el ámbito de polifenoles y sus aplicaciones industriales, productos innovadores con microalgas, etc.), así como centrados en determinadas infraestructuras (tratamiento de los vertidos industriales y domésticos, un sistema de vigilancia ambiental para su utilización en redes de

99. UE, Interreg Europe, op. cit.

100. UE, Interreg Europe, op. cit.

101. Unión Europea, Programa Cooperación Transfronteriza España-Portugal. En línea en: http://www. poctep.eu/es. [Consulta: 18.06.18].

102. Red de Iniciativas Urbanas, “132 proyectos aprobados para el POCTEP 2014-2020”. En línea en: http://www.rediniciativasurbanas.es/novedades-y-eventos/noticias/170509-0. [Consulta: 18.06.18].

103. Junta de Extremadura, Programa Interreg V-A España-Portugal (POCTEP) 2014-2020. En línea en: http://www.juntaex.es/filescms/ddgg006/uploaded_files/DDGG_POLITICA/Actualidad_Economica/ Interreg_V-A-1.pdf. [Consulta: 18.06.18]. 
alerta temprana, etc.) o equipamientos (un observatorio costero en el SO ibérico; un instituto de bioingeniería en red, etc. $)^{104}$.

Para lograr altos niveles de integración, se requiere de actores políticos y económicos decididos a emprender proyectos con los vecinos del otro lado de la frontera y una sociedad que entienda la cooperación y el entendimiento ofrece mejores frutos que el enfrentamiento y la incomunicación ${ }^{105}$. Por ello, son necesarios programas de auténtica cooperación transfronteriza principalmente en los ámbitos de la co-gobernanza, la movilidad y el medio ambiente. No parece que dar subvenciones más o menos cuantiosas a más de un centenar de proyectos distintos, que van desde la protección del lince ibérico hasta el patrimonio cultural en común ${ }^{106}$, sea la metodología más conveniente. Por mucho que sean loables los objetivos de estos 132 proyectos (301 fueron denegados ${ }^{107}$ ), una planificación estratégica de cooperación transfronteriza basada en la co-gobernanza y con un diseño multisectorial y amplio es una opción más racional para futuros programas POPTEC, o similares.

Por otro lado, superada la fase más oscura de la recesión de 2008, se fortalece en el presente el papel de una figura creada en el 2006 dentro del marco de la Cooperación Territorial Europea. Son las Agrupaciones Europeas de Cooperación Territorial (AECTs). Su importancia está en las ventajas que aporta con respecto a las normativas existentes. Expertos en Derecho comunitario nos señalan que «supone un salto cualitativo en la creación de organismos de cooperación entre entidades territoriales de muy diversa índole, incluyendo a Estados, y despeja dudas sobre la naturaleza jurídica de estos organismos que en ningún caso estarán regidos por el derecho internacional público» ${ }^{108}$. En resumen, dotan a las entidades territoriales de una herramienta institucional para la cooperación transfronteriza, transnacional e interregional ${ }^{109}$. Con participación española y portuguesa pronto se constituyeron AECTs de cooperación ${ }^{110}$.

Dentro de este nuevo impulso a la cooperación transfronteriza, la XXIX Cumbre LusoEspañola celebrada en mayo de 2017 en la ciudad portuguesa de Vila Real mostró una clara voluntad de recuperar el tiempo perdido. Por primera vez, la Cumbre ha centrado sus trabajos específicamente en la cooperación transfronteriza y, especialmente, en sus aspectos eco-

104. Unión Europea, Programa Cooperación..., op. cit.

105. Trillo y Lois, “La frontera..., op. cit., 129.

106. Unión Europea, Programa Cooperación..., op. cit.

107. Red de Iniciativas Urbanas, “132 proyectos...”, op. cit.

108. S. Beltrán García, "La cooperación transfronteriza e interterritorial: un clásico renovado”, Revista d'EstudisAutonòmics i Federals, 4, 2007, 215-246.

109. S. Beltrán García, "Qué esperar de la figura de la Agrupación Europea de Cooperación Territorial (AECT) en relación con los organismos creados de cooperación creados por las Comunidades Autónomas", Revista General de Derecho Europeo, 14, 2007, 1-35.

110. E. J. Martínez Pérez, "Las Agrupaciones Europeas de Cooperación Territorial (Unión Europea) frente a las Agrupaciones Eurorregionales de Cooperación (Consejo de Europa): ¿competencia o complementariedad?", Revista de Estudios Europeos, 56, 2010, 109-126. 
nómicos, medioambiental, energético y de infraestructuras ${ }^{111}$. De hecho, como resultado se ha publicado una declaración conjunta con un elevado número de puntos que son compromisos para unas futuras estrechas relaciones bilaterales. Por ejemplo, entre los puntos 14 y 24 se suscribe una «Declaración de Intenciones sobre Transportes e Infraestructuras de Transportes Transfronterizas» que pretende recuperar la inversión económica en las redes ferroviarias o mejorar el sistema exclusivamente electrónico de peaje para usuarios extranjeros de Portugal, entre otras medidas ${ }^{112}$. No obstante, ¿cuál será la aplicación real de los acuerdos? Se plantean dudas cuando uno de los firmantes, el Presidente del Gobierno de España, ha sido sustituido tras la primera moción de censura efectiva de la democracia española en 2018.

\section{Nuevos escenarios de cooperación en el marco de la UE y en el de las relaciones transfronterizas}

En el presente, avanzado el siglo XXI, se plantean para España y Portugal nuevos escenarios futuros de cooperación en el marco de la UE. La construcción de entidades de gestión conjuntas a partir de las citadas AECTs supone un marco jurídico ventajoso para avanzar en la cooperación interregional transfronteriza. Se trata de nuevos centros de poder transfronterizo. Los ámbitos de actuación de las AECTs son muy amplios y posibilitan dos vías de desarrollo: la primera, mediante la aplicación de programas de cooperación territorial cofinanciados por la UE con cargo a los fondos estructurales aprobados; y la segunda, a través de iniciativas propias de cooperación territorial, esto es, impulsadas por los miembros de la AECT sin intervención de la UE ${ }^{113}$.

Entre los lugares de integración que están surgiendo y deben consolidarse en el futuro, destaca el papel fundamental de la cooperación en $\mathrm{I}+\mathrm{D}+\mathrm{i}$ de contenido transnacional. Esta colaboración se traduce en un incremento de la capacidad competitiva y de la presencia exterior de los territorios ibéricos ${ }^{114}$. Un ejemplo importante es el Centro Ibérico de Nanotecnología de Braga. Se trata de un campus de $47.000 \mathrm{~m}^{2}$ localizado próximo a la Universidade do Minho. Un instituto intergubernamental español-portugués es el encargado de fomentar la investigación interdisciplinar con aplicaciones en la bioingeniería, los biomateriales y la nanomedicina, entre otras ${ }^{115}$.

111. Gobierno de España, “Declaración conjunta de la XXIX Cumbre Luso-Española”. En línea en: http:// www.lamoncloa.gob.es/presidente/actividades/Documents/2017/Declaracio\%CC\%81n\%20Conjunta\%20 XXIX\%20Cumbre\%20PT-ES\%20(26-05-2017)_ES.pdf. [Consulta: 19.06.18].

112. op. cit.

113. Beltrán, “La cooperación transfronteriza..., op. cit., 230.

114. Lois y Carballo, "La frontera hispano-lusa..., op. cit., 214.

115. Centro de Investigación Biomédica en Red en el área temática de Bioingeniería, Biomateriales y Nanomedicina (CIBER-BBN), Laboratorio Internacional de Nanotecnología. Braga, Portugal. En línea en: http://www.ciber-bbn.es/plataformas/plataformas-asociadas/laboratorio-internacional-de-nanotecnologia-inl-braga-portugal. [Consulta: 18.06.18]; InvestBraga, Laboratório Ibérico internacional de Nanotecno- 
Otra interesante experiencia es la Fundación Centro de Estudios Eurorregionales Galicia-Norte de Portugal (CEER). Su objetivo principal es la búsqueda de sinergias y complementariedades entre los centros universitarios de Galicia-Norte de Portugal ${ }^{116}$. Entre sus líneas básicas de actuación destaca la promoción, dinamización y desarrollo de la investigación interuniversitaria de carácter multidisciplinar en la eurorregión ${ }^{117}$.

Por otro lado, se ha fortalecido una integración empresarial transfronteriza. Un ejemplo destacado es el de la pesca y la industria asociada por los notables intereses y negocios comunes que se fueron densificando entre sí y también con Cabo Verde, Guinea-Bissau, Angola y Mozambique ${ }^{118}$. También es significativo el caso de la industria automovilística. El grupo PSA está instalado desde hace más de medio siglo en la ciudad gallega de Vigo y en la de Mangualde en la Región Centro de Portugal. PSA ha aprovechado las oportunidades de cooperación transfronteriza para acceder con mayor facilidad y de manera conjunta a los mercados español y portugués y para adaptar mejor sus estrategias empresariales ${ }^{119}$. Otras compañías, dentro del sector de la logística, han considerado la proximidad de la frontera como un factor interesante para el desarrollo de sus actividades (en la aglomeración de Vigo, alrededor del Miño, en Viana do Castelo, etc. ${ }^{120}$. Y, desde la Región Norte lusa, empresas del sector textil y calzado y del de la madera y mueble elaboran manufacturas o venden en relación con compañías españolas ${ }^{121}$. Son sinergias que derivan en zonas de localización empresarial transfronteriza ${ }^{122}$.

Al mismo tiempo, hoy se han conformado ejes urbanos de desarrollo entre Galicia y la Región Norte portuguesa y en el litoral onubense-algarví. Los flujos e intercambios de bienes, personas y servicios permiten hablar de una vida transfronteriza común interurbana. El eje atlántico desde Setúbal hasta A Coruña-Ferrol define el corredor de mayor concentración urbana y económica de la Península Ibérica ${ }^{123}$. Se plantea para el futuro un paso siguiente de fortalecimiento y complejidad urbana, la posible formación de conurbaciones hispanoportuguesas. En este sentido, la relación y cooperación entre Oporto y Vigo debe convertirse en una prioridad ${ }^{124}$. Son las dos ciudades más pobladas e industrializadas del eje atlántico. En

logia. En línea en: https://www.investbraga.com/knowledge/international-iberian-nanotechnology-laboratory. [Consulta: 18.06.18].

116. Centro de Estudios Euro-Regionales Galicia-Norte de Portugal. En línea en: http://www.fceer.org/ noticias/activos/1223542361-Presentacion_CAS.pdf. [Consulta: 19.06.18].

117. op. cit.

118. Lois, “Galiza no espaço lusófono..., op. cit., 13.

119. Agencia Efe, "PSA producirá en Vigo y Portugal una nueva generación de furgonetas para el ocio", 2018. En línea en: https://www.eldiario.es/economia/PSA-Vigo-Portugal-generacion-furgonetas_0_739826188.html. [Consulta:14.06.18].

120. Lois, "Galice-Portugal..., op. cit., 36.

121. Trillo y Lois, “La frontera..., op. cit., 127.

122. Trillo y Lois, "La frontera..., op. cit., 127.

123. Lois, “Galiza no espaço lusófono...”, op. cit., 9.

124. op. cit. Lois, “Galiza no espaço lusófono...”, op. cit., 
el sur de la Península Ibérica, la creciente interrelación comercial, industrial y portuaria entre las ciudades medias de Huelva y Faro sería otra posible conurbación ${ }^{125}$.

En otros campos de clara cooperación común, un necesario avance se plantea como inaplazable. Es el caso de un grave problema ambiental como son los incendios forestales. Esta «plaga» afecta anualmente a ambos espacios de la frontera, a veces, como en el caso del verano de 2017, con elevados costes materiales y, lamentablemente, humanos ${ }^{126}$. Las consecuencias catastróficas y los problemas de degradación ambiental obligan a pensar y poner en marcha nuevos modelos de gobernanza conjunta de los espacios naturales de interés ${ }^{127}$. Es el ejemplo, también, de la cosoberanía de los territorios fluviales. España y Portugal comparten varias de las cuencas hidrográficas principales de la Península Ibérica: Miño, Duero, Tajo y Guadiana. De hecho, el Miño y el Guadiana delimitan parte de la frontera, la raya húmeda, la más densa demográficamente, la más urbanizada y la más dinámica económicamente. La gestión común de estas cuencas hidrográficas es la solución lógica desde el punto de vista ambiental. Sin embargo, la explotación humana y económica del agua es una cuestión conflictiva, muy complicada de plantear en una gobernanza transfronteriza común. No obstante, la cuestión medioambiental no conoce de fronteras políticas. La conservación y gestión de ecosistemas acuáticos es responsabilidad de toda la comunidad y precisa de una cooperación entre España y Portugal para lograr la sostenibilidad del medio natural ${ }^{128}$.

Una realidad todavía no resulta es la situación de la denominada raya seca ${ }^{129}$. Este territorio incluye espacios rurales de interior de Castilla y Extremadura, en España, y de TrásOs-Montes, la Beira interior y el Alentejo, en Portugal. Forma una de las áreas más pobres, envejecidas y despobladas de la Península Ibérica ${ }^{130}$. Hay excepciones como el eje BadajozElvas $^{131}$ o el corredor transfronterizo Chaves-Verín ${ }^{132}$. Sin embargo, mayoritariamente se trata de comarcas con un perfil económico bastante débil donde una escasa industrialización se concentra en muy limitadas cabeceras. La agricultura y ganadería se especializan en algunos productos agrarios de calidad, pero el contexto general es de crisis y abandono agropecuario. Además, la edad media de la población es muy elevada, todavía más en los territorios españoles que en los portugueses ${ }^{133}$. Las políticas de desarrollo regional y la cooperación trans-

125. J. A. Márquez Domínguez, "El eje dinámico del litoral onubense-algarví”, en L. López, R.C. Lois y F. Guichard (Coords.), La articulación territorial de la raya hispano-portuguesa, Zamora, 1997, 143-149.

126. J. Martín del Barrio, “¿Por qué los incendios en Portugal son tan letales?”. En línea en: https://elpais. com/internacional/2017/10/19/actualidad/1508410409_311317.html. [Consulta: 18.06.18].

127. Lois y Carballo, “La frontera hispano-lusa..., op. cit., 214.

128.TeamMinho, "Transferencia de herramientas para la evaluación, ordenación, gestión y educación ambiental en estuarios”. En línea en: http://www.team-minho.eu/index.php/es/. [Consulta: 18.06.18].

129. Lois, “As relaçons..., op. cit., 197.

130. Lois y Plaza, “Development policies...”, op. cit., 317.

131. J. A. Gutiérrez Gallego y J. M. Pérez Pintor, "Nuevas dinámicas territoriales en los espacios fronterizos hispano-lusos: el caso de las ciudades de Elvas y Badajoz", en I. Pujadas et al. (Coords.), Población y espacios urbanos: XII Congreso de Población Española, Barcelona, 2011, 127-142.

132. L. Domínguez Castro (Coord.), Chaves-Verín. A eurocidade da Auga. Axenda Estratéxica, Vigo, 2008.

133. Lois y Carballo, “La frontera hispano-lusa..., op. cit., 208-212.

Revista de historiografía 30, 2019, pp. 157-180 
fronteriza de la UE han incidido positivamente con la mejora de infraestructuras y las ayudas para el emprendimiento económico. No obstante, la situación desfavorecida en este espacio debe marcar en el futuro una continuidad en las estrategias de desarrollo local. Igualmente, dada la realidad demográfica de la raya seca, son inaplazables políticas de envejecimiento activo $^{134}$.

\section{Una cuestión mayor: frontera o fronteras en un mundo de escalas cambiantes}

Desde el año 1986, con la integración de España y Portugal en la entonces CEE y, fundamentalmente, desde 1992 con la constitución de la actual UE se produce un proceso creciente de des-fronterización ibérica, como ya se ha explicado. Sin embargo, al tiempo que desaparecían las fronteras internas, se ha producido una creciente re-fronterización hacia el exterior. La Península Ibérica asume ahora un rol fundamental derivado de su localización geográfica más básica: es parte de la frontera exterior de la UE. Una frontera principalmente marítima, bien es cierto, pero también terrestre en las ciudades autónomas de Ceuta y Melilla, que hoy demuestra ser sumamente atractiva para las migrantes procedentes del Sur Global.

De esta forma, el Parlamento Europeo ha adoptado un nuevo sistema de vigilancia de las fronteras exteriores de la UE conocido como «Eurosur». Se trata de un sistema paneuropeo de vigilancia fronteriza en red que busca mejorar la detección, prevención y lucha contra la inmigración irregular y la delincuencia organizada ${ }^{135}$. España y Portugal forman parte de esta red. De hecho, en la indicada cumbre de Vila Real se acordó la interconexión del Sistema Integrado de Vigilancia y Control Costero (SIVICC) de Portugal con el Sistema Integrado de Vigilancia Exterior (SIVE) $)^{136}$.

Estas medidas adoptadas forman parte de las estrategias de la Agencia Europea de la Guardia de Fronteras y Costas (FRONTEX). Creada en 2004, ayuda a los Estados miembros de la UE y a los países asociados al espacio Schengen (Islandia, Noruega y Suiza) a gestionar sus fronteras exteriores y facilita la cooperación entre las autoridades fronterizas de cada país de la UE, ofreciendo apoyo técnico y experiencia ${ }^{137}$. ¿Por qué en un organismo que impulsa la integración supranacional ha sido necesario crear una agencia ex novo de control fronterizo común? La respuesta que proporciona la propia agencia es lo suficientemente explícita y explicativa: «en los últimos años, las fronteras exteriores de Europa han visto un aumento sin precedentes del número de refugiados y migrantes que desean entrar en la UE. Los paí-

134. P. Palomino Saura, "Medidas para el fomento del envejecimiento activo", Nueva Revista Española de Derecho del Trabajo, 162, 161-176.

135. Parlamento Europeo, "Eurosur: el PE adopta un nuevo sistema de vigilancia de las fronteras exteriores”. En línea en: http://www.europarl.europa.eu/news/es/press-room/20131007IPR21624/eurosur-el-peadopta-un-nuevo-sistema-de-vigilancia-de-las-fronteras-exteriores. [Consulta: 19.06.18].

136. Gobierno de España, "Declaración conjunta...", op. cit.

137. Unión Europea, Agencia Europea de la Guardia de Fronteras y Costas (FRONTEX). En línea en: https://europa.eu/european-union/about-eu/agencies/frontex_es\#overview. [Consulta: 19.6.18]. 
ses con fronteras exteriores son responsables exclusivos de su control. Sin embargo, Frontex puede proporcionar apoyo técnico adicional a los países de la UE que sufren graves presiones migratorias» ${ }^{138}$.

Al tiempo que se refuerzan las fronteras exteriores de la UE, por lo tanto, una re-fronterización, el proceso contrario, el de la des-fronterización ya ha avanzado significativamente. Son ejemplos destacados de integración las regiones transfronterizas o eurorregiones, como la de Galicia-Región Norte y la del Alentejo-Algarve-Andalucía. Las eurorregiones han hecho posible un acercamiento a escala regional de carácter cultural, económico, institucional, social y territorial. Igualmente, un resultado de la des-fronterización ha sido la emergencia de un eje urbano atlántico que integra una urbanización continua gallega (desde Ferrol y A Coruña hasta Vigo) y continua por el litoral portugués (Viana do Castelo-Braga-GuimarãesOporto-Aveiro-Lisboa-Setúbal) $)^{139}$.

No obstante, y siguiendo con un análisis dialéctico del proceso, también se puede afirmar que entre los Estados-nación ibéricos aún persisten importantes desencuentros. Sin duda, un problema mayor son los contrastes en el mapa político de España y Portugal. España es un país fuertemente descentralizado desde la aprobación de la Constitución de 1978. Portugal es un Estado muy centralista donde no existen autoridades regionales directamente elegidas. De esta manera, se confrontan dos realidades distintas, una, la española, con una elevada autonomía de las distintas comunidades, y la otra, la portuguesa, donde después del rechazo del referéndum de 1998 el proyecto de regionalización pasa por un largo período de «hibernación» ${ }^{140}$. En este contexto, se presentan dificultades prácticas en las relaciones transfronterizas entre las administraciones. Las comunidades autónomas españolas dialogan con regiones portuguesas sin apenas competencias y capacidad de decisión. La administración central portuguesa se encuentra con que su homónima española se presenta descentralizada.

Pese a estas dificultades, la desaparición de la frontera ha conllevado unos beneficiosos efectos económicos y socioculturales. Por esta razón, el viejo sueño del iberismo de hace más de un siglo ${ }^{141}$, entendido como una unidad política federal o confederal de la Península, se puede reinterpretar hoy bajo la lógica de integración de territorios y países limítrofes impulsada por la UE. A este respecto, las complementariedades económicas, la vecindad, la facilidad de comunicaciones, una organización tradicional del espacio semejante y la simpatía existente entre las dos comunidades ayuda a una creciente integración ${ }^{142}$. No obstante, ciertas realidades fronterizas permanecen como el contraste entre el mapa político de España y Portugal. Además, socialmente es obvio que las identidades nacionales siguen estando alejadas. La crisis de 2008 supuso, a mayores, un grave freno al creciente proceso de cooperación hispano-portuguesa. Desde el año 2014, superados sus efectos más nocivos, las relaciones

138. op. cit.

139. Rodríguez et al., "El eixo atlántico...”, op. cit., 123-136.

140. J. López-Davalillo, "Portugal, Qué Regionalización? Un recorrido por la geografía política de Portugal a lo largo del tiempo", Espacio, tiempo y forma. Serie VI, Geografía,3, 2012, 27-52.

141. Rocamora, “Un nacionalismo fracasado..., op. cit.

142. Lois, "Galiza no espaço lusófono...”, op. cit., 31.

Revista de historiografía 30, 2019, pp. 157-180 
transfronterizas se recuperan vigorosamente, pero el camino hacia la integración, donde dos suman más que uno ${ }^{143}$, aún es largo.

\section{Conclusión}

A lo largo del presente artículo hemos procurado revisar teóricamente el estudio del hecho fronterizo, analizando en particular las relaciones y la raya hispano-portuguesa. En la misma, el proceso de des-fronterización avanza a un ritmo desigual dependiendo del impulso europeo a la cooperación y de la situación económica (y política) del momento en los dos Estados-nación. Desde el 1986, se ha progresado considerablemente en la cooperación ibérica, pero algunos desajustes estructurales no han podido superarse y, sin duda lo más importante, el impulso a la cooperación muestra síntomas de fragilidad, presenta interrogantes sobre su sostenibilidad en el tiempo. Las buenas relaciones hispano-portuguesas y las instituciones transfronterizas son hechos consolidados, ahora se trataría de avanzar en arquitecturas de gobernanza conjuntas (a través de la figura de las AECTs) y en el mantenimiento de fondos permanentes de cooperación no sujetos a las coyunturas.

En la Estrategia Europea de 2020 se ha insistido en favorecer el crecimiento inteligente, sostenible e integrador. Estos tres aspectos han caracterizado las sinergias en la cooperación transfronteriza, que funcionan francamente bien en el caso de Galicia y la Región Norte de Portugal ${ }^{144}$, de modo aceptable en Andalucía-Algarve y Extremadura-Alentejo, y con menor fuerza en otras partes de la raya, como en Castilla y León. Las relaciones fronterizas, superada la peor fase de la recesión económica global de 2008, responden a las políticas de desarrollo interregional que vienen marcando desde la década de 1990 objetivos principales dentro de la planificación e intenciones de la UE. En el futuro, a punto de acabarse el marco temporal de 2020, avanzar en el mismo sentido ha de ser prioritario.

Como apunte final, cabe recordar que la salida de la crisis económica de Portugal y España ha sido ejemplar durante estos últimos años. Quizás sería el momento de reivindicar este ejemplo de superación de las graves dificultades financieras, al tiempo que se avanza en la idea de reforzar un lobby ibérico en las instituciones europeas.

143. Lois, “Galiza no espaço lusófono..., op. cit., 31.

144. Lois, “Galiza no espaço lusófono..., op. cit., 20. 